\title{
KRAS Mutation as a Biomarker for Survival in Patients with Non-Small Cell Lung Cancer, A Meta-Analysis of 12 Randomized Trials
}

\author{
Min Ying ${ }^{1 \&}$, Xiao-Xia Zhu ${ }^{1 \& *}$, Yang Zhao ${ }^{2}$, Dian-He $\mathrm{Li}^{1}$, Long-Hua Chen ${ }^{1}$
}

\begin{abstract}
Background: Because there is no clear consensus for the prognostic implication of KRAS mutations in patients with non-small cell lung cancer (NSCLC), we conducted a meta-analysis based on 12 randomized trials to draw a more accurate conclusion. Materials and Methods: A systematic computer search of articles from inception to May 1, 2014 using the PubMed, EMBASE, and Cochrane databases was conducted. The enrollment of articles and extraction of data were independently performed by two authors. Results: Our analysis was based on the endpoints overall survival (OS) and progression-free survival (PFS). Nine records (All for OS, 7 for PFS) comprising 12 randomized trials were identified with 3701 patients who underwent a test for KRAS mutations. In the analysis of the pooled hazard ratios (HRs) for OS (HR: 1.39; 95\% confidence interval [CI] 1.23-1.56) and PFS (HR: 1.33; 95\% CI 1.17-1.51), we found that KRAS mutations are related to poor survival benefit for NSCLC. According to a subgroup analysis stratified by disease stage and line of therapy, the combined HRs for OS and PFS coincided with the finding that the presence of a KRAS mutation is a dismal prognostic factor. However, the prognostic role of KRAS mutations are not statistically significant in a subgroup analysis of patients treated with chemotherapy in combination with cetuximab based on the endpoints $O S(P=0.141)$ and PFS $(P=0.643)$. Conclusions: Our results indicate that KRAS mutations are associated with inferior survival benefits for NSCLC but not for those treated with chemotherapies integrating cetuximab.
\end{abstract}

Keywords: KRAS - non-small cell lung cancer - survival - meta-analysis

Asian Pac J Cancer Prev, 16 (10), 4439-4445

\section{Introduction}

Although consistent progress has been made in diagnostic methods and treatment regimens, lung cancer remains the leading cause of cancer deaths worldwide with non-small cell lung cancer (NSCLC) accounting for approximately $85 \%$ of lung cancer patients (Ramalingam et al., 2011). Currently, many NSCLC patients present with an advanced stage at diagnosis, which may be partially due to non-typical early symptoms, lymphatic metastasis and hematogenous metastasis. Platinumbased cytotoxic chemotherapy, which was the standard therapy for advanced NSCLC has been considered to reach an efficacy plateau, whereas the targeted treatment of epidermal growth factor has provided a new insight for future personalized therapy treatment strategies (Carney, 2002). Until now, epidermal growth factor receptor (EGFR) mutation was identified to be a favorable predictive factor for EGFR tyrosine kinase inhibitor (TKI) treatment response (Usuda et al., 2014) but not a prognostic marker (NCCN, 2012), and EGFR-TKIs revealed encouraging efficacy, safety and survival for not only maintenance therapy but also second line therapy for advanced NSCLC (Qi et al., 2012; Alimujiang et al., 2013), which suggested avenues for future studies on the identification of additional potential biomarkers.

The KRAS oncogene is involved in tumorigenesis and encodes membrane-bound 21-kd proteins with an intrinsic guanosine triphosphatase (GTPase), which was noted for decades (Santos et al., 1984). Activation of KRAS proteins is triggered by extracellular stimuli, resulting in a switch from a guanosine diphosphate (GDP)-bound form of KRAS to a guanosine triphosphate (GTP)-bound form (Martin et al., 2013). The presence of a KRAS mutation in a wide variety of cancers (Chetty and Govender, 2013) was reported to be the most common molecular change in NSCLC, with an average mutation rate of $20-30 \%$ in adenocarcinoma and only rare mutations in squamous tumors, leading to the cascade activation of downstream effectors and cell proliferation (Roberts and Stinchcombe, 2014). In lung cancer, greater than $95 \%$ of KRAS mutations present in codons 12 and 13 (Riely et al., 2008).

Many clinical studies have attempted to determine a prognostic role for KRAS mutations; however, controversial conclusions persist because the first report indicated that a $K R A S$ codon 12 point mutation was related

${ }^{1}$ Department of Radiation Oncology, ${ }^{2}$ The First Clinical Medical College, Nanfang Hospital, Southern Medical University, Guangzhou, China ${ }^{\star}$ Equal contributors *For correspondence: zhuxx01@126.com 
to unfavorable prognosis based on the survival analysis of 69 patients with cancers ranging from stage I to stage IIIa (Slebos et al., 1990). While in another retrospective study on the implication of KRAS mutations, which was also analyzed in a cohort of patients with surgically resected early-stage NSCLC, KRAS mutation was a negative prognostic factor only for a stage II NSCLC subgroup but not the entire group comprising stage I and stage II NSCLC (Graziano et al., 1999). In addition, in the NCIC CTG BR 19 study, no statistically significant relationship was noted between $K R A S$ mutation and disease-free survival (DFS) or overall survival (OS) for the patients with completely resected tumors (Goss et al., 2013). For advanced NSCLC, there is also no unequivocal conclusion. In a randomized trial investigating the efficacy of erlotinib as a maintenance treatment, which was reported by Brugger, KRAS mutation emerged as a significant poor prognostic factor for progression-free survival (PFS) in the placebo arm, revealing that KRAS mutation is related to unfavorable survival regardless of therapy (Brugger et al., 2011). Nevertheless, there are also studies that found that KRAS mutation is related to shorter survival for patients with advanced NSCLC (O'Byrne et al., 2011, Johnson et al., 2013). To resolve these differences, newer and larger meta-analyses are needed.

With regards to the most likely limited small sample sets and considerable heterogeneity among studies including different lines of treatment and enrollment of patient populations, we conducted an up-to-date metaanalysis based on 9 publications including patients from 12 randomized trials to fully assess the prognostic role of $K R A S$ mutations in a group of NSCLC patients and further explore the implications of $K R A S$ mutation in a specific subgroup of NSCLC patients.

\section{Materials and Methods}

\section{Identification of eligible trials}

After generating a search strategy, we performed a comprehensive systematic search in the PubMed, EMBASE and the Cochrane library databases from inception to May 1, 2014 using the following keywords: "non-small cell lung cancer," "non-small cell lung carcinoma," "non-small cell lung neoplasm," "nonsmall cell lung tumor," "non-small cell lung tumour," "NSCLC," "pulmonary adenocarcinoma," "lung adenocarcinoma," "adenocarcinoma of the lung," "lung squamous carcinoma," "pulmonary squamous carcinoma," "squamous cell lung carcinoma," "squamous carcinoma of the lung," "KRAS," "k-ras," "ki-ras," "random" and "randomized". Bibliographies from related meta-analyses or reviews were also searched. In addition, we also managed to contact corresponding authors for additional unpublished data. Abstracts or meeting proceedings were excluded.

\section{Selection criteria}

Acquired related citations in the form of abstracts were independently assessed by two authors according to the following exclusion criteria: (i) non-randomized trials and (ii) studies performed with NSCLC cell lines or animal models. Then, further screening of studies was performed with the following additional inclusion criteria: (i) trials that were published and written in English, (ii) randomized studies on the prognostic role of KRAS mutations in NSCLC patients without treatment restriction, and (iii) adequate data for estimating hazard ratios (HRs) and their $95 \%$ confidence intervals (CIs) for OS and PFS stratified by KRAS mutation status. Articles that could not be decisively excluded according to their abstracts were further assessed by searching for the full articles and corresponding supplementary data. With regards to studies with overlapping patient populations, the most recent investigation using updated data for publication was chosen for inclusion.

\section{Data extraction}

The following information was extracted based on the Preferred Reporting Items for Systematic Reviews and Meta-analyses statement (Liberati et al., 2009) from each recovered article: first author, year of publication, number of patients enrolled in the study, number of patients who were assessed for KRAS mutation status, median age, number of patients with a KRAS mutation, treatment regimen, primary endpoint, codon analyzed, demographic data (i.e., number of females, smoking history, stage of disease, and histology), and data linking $K R A S$ mutation to treatment outcome (i.e., HR). Data extraction was conducted independently by two authors (MY and YZ) in accordance with a predefined information sheet. Discrepancies were discussed to reach consensus by including a third author.

\section{Statistical analysis}

For the purpose of analysis, the primary outcome was OS and the secondary outcome was PFS, which were expressed as a HR with $95 \%$ confidence interval (CI) for every treatment arm stratified by KRAS oncogene status. OS was defined as the duration of survival from randomization. PFS was determined as the time from randomization to progression, recurrence, of death from any cause or the last follow-up during the trial period, which was under the assumption that PFS may not be different from time to progression. When HRs comparing mutant with wildtype $K R A S$ patients was not directly reported in a study, three independent investigators extracted basic survival data from the form of graphical curves using Engauge Digitizer version 4.1. Individual HRs and their variance in each treatment arm were reconstructed according to the method reported by Tierney et al. (2007). If the HR indicated comparisons between wildtype and mutant $K R A S$ patients, reciprocals of both the HR and its variance were extracted for approximate estimation. These HR estimates were then combined to give an overall HR. An observed, a HR above 1 indicated that poorer outcome was associated with $K R A S$ mutation.

Heterogeneity was determined by the Chi-square and $I^{2}$ tests $\left(I^{2}<25 \%\right.$ no heterogeneity; $I^{2}=25-50 \%$ moderate heterogeneity; $I^{2}>50 \%$ substantial heterogeneity) (Higgins et al., 2003). All analyses were first conducted using the Mantel-Haenazel fixed mode (Mantel and Haenszel, 1959) based on the assumption of no extreme 
heterogeneity $\left(I^{2} \leq 50 \%\right)$. The DerSimonian-Laird random model (DerSimonian and Laird, 1986) was then used if rejected. Tests were considered statistically significant if $P$ was $<0.05$ with the exception of the heterogeneity test. All statistical tests were conducted with STATA version 12.0 (Stata Corporation, TX and USA).

\section{Results}

Study selection and characteristics

A total of 637 potentially relevant articles were identified from three databases, and 403 articles were excluded for duplication as indicated in the flow chart of study selection (Figure 1). After screening titles and abstracts following the exclusion criteria, 92 articles were enrolled for further eligibility assessment, and 9 records (Schiller et al., 2001; Eberhard et al., 2005; Zhu et al., 2008; Douillard et al., 2010; Herbst et al., 2010; Khambata-Ford et al., 2010; Brugger et al., 2011; O’Byrne et al., 2011; Shepherd et al., 2013) were ultimately used in the meta-analysis. The characteristics of these 9 records are summarized in Table 1. Because the outcomes reported by Shepherd published in 2013 used individual data from 4 randomized trials including IALT (International Adjuvant Lung Cancer Trial), ANITA (Adjuvant Navelbine International Trialist Association), JBR.10 and Cancer and Leukemia Group B (CALGB) 9633, the final analysis covered data from patients from 12 randomized controlled trials in total. There were 10210 total NSCLC patients in all studies, and 3701 patients were available for testing for $K R A S$ mutations with 695 patients $(18.8 \%)$ harboring a mutation. Six studies (Eberhard et al., 2005; Schiller et al., 2001; Herbst et al., 2010; Khambata et al., 2010; O'Byrne et al., 2011; Shepherd et al., 2013) were performed in the first-line setting, and two (Zhu et al., 2008; Douillard et al., 2010) had second-line or higher treatment with one (Brugger et al., 2011) investigating maintenance treatment with erlotinib in nonprogressive patients after first-line platinum-based chemotherapy. All trials with the exception of two (Schiller et al., 2001, Shepherd et al., 2013) were conducted with advanced NSCLC patients. When stratifying by treatment regimen, chemotherapy was set as a treatment arm in five publications (Eberhard et al., 2005, Douillard et al., 2010, Khambata-Ford et al., 2010; O'Byrne et al., 2011; Shepherd et al., 2013), EGFR-TKIs in two articles (Zhu et al., 2008, Douillard et al., 2010) and the addition of cetuximab to chemotherapy in three articles (Herbst et al., 2010; Khambata-Ford et al., 2010; O’Byrne et al., 2011).

Association between KRAS mutations and survival benefit All studies, including 646 patients with a KRAS mutation and 2699 patients with wildtype $K R A S$ and the samples ranging from 39 to 780 patients per treatment arm, were used for OS analysis. Results from the pooled model indicated that significant poor survival benefit was associated with KRAS mutation in NSCLC patients (HR: $1.39 ; 95 \%$ CI: $1.23-1.56 ; P<0.001$ ) (Figure 2A). No significant heterogeneity between the studies was noted $\left(I^{2}=33.9 \% ; \mathrm{P}=0.091\right)$. The 7 studies (Eberhard et al., 2005,

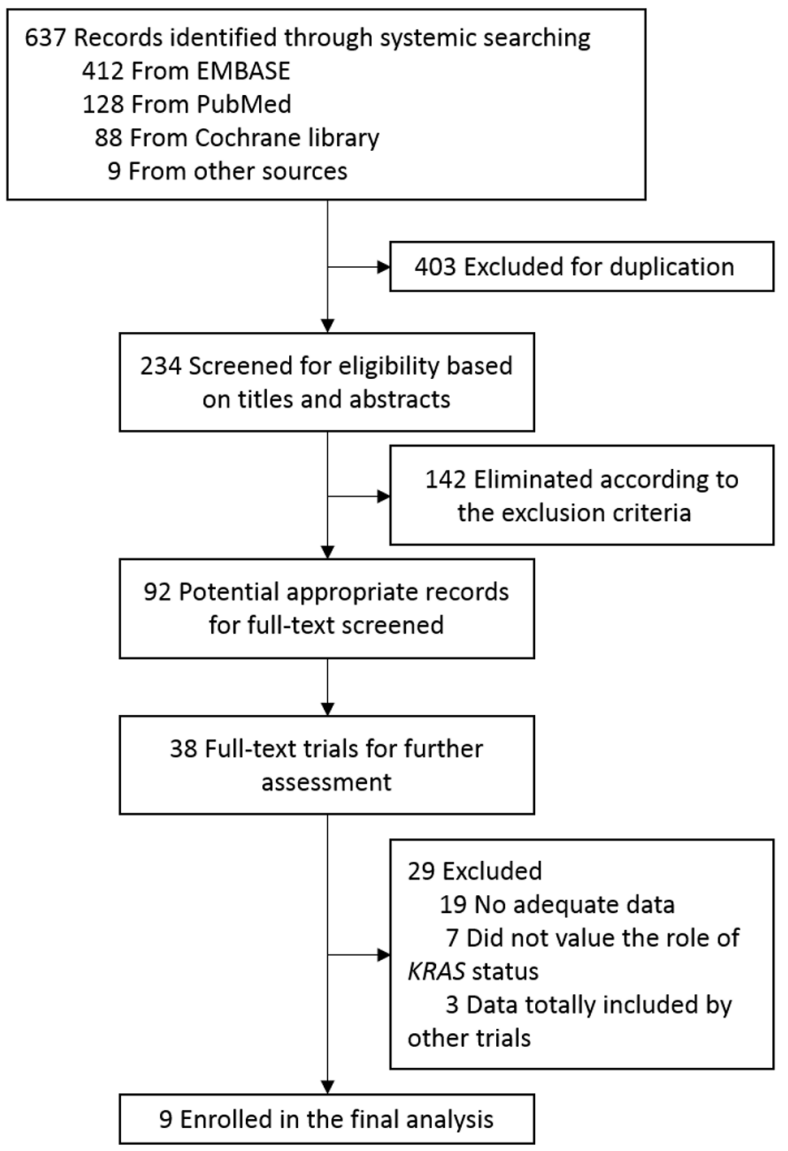

Figure 1. Flow Chart of the Selection Process of Studies

Table 1. Baseline Characteristics of the Included Studies on the Prognostic role of KRAS in NSCLC

\begin{tabular}{|c|c|c|c|c|c|c|c|c|c|c|c|c|}
\hline First author & r Year & $\begin{array}{l}\text { Patients } \\
\text { enrolled }\end{array}$ & $\begin{array}{c}K R A S \\
\text { assesed }\end{array}$ & $\begin{array}{c}\text { Median } \\
\text { age, Y }\end{array}$ & $\begin{array}{c}\text { Mut } K R A S \\
(\%)\end{array}$ & $\begin{array}{c}S \text { Female } \\
(\%)\end{array}$ & $\begin{array}{c}\text { Never } \\
\text { smoker }(\%)\end{array}$ & Stage & $\begin{array}{c}\text { Adeno-ca } \\
(\%)\end{array}$ & Treatment & $\begin{array}{l}\text { Primary } \\
\text { endpoint }\end{array}$ & $\begin{array}{c}\text { Codon } \\
\text { t analyzed }\end{array}$ \\
\hline Schiller & 2001 & 488 & 184 & 60.5 & $44(23.9)$ & 203 (41.6) & NA & II-IIIA & $259(53.1)$ & $\mathrm{RT}+\mathrm{CisE}$ vs RT & OS & $12,13,61$ \\
\hline Eberhard & 2005 & 1079 & 271 & 62.6 & $55(20)$ & 424 (39.3) & $116(10.8)$ & IIIB-IV & $654(60.6)$ & $\mathrm{CP}+\mathrm{E}$ vs $\mathrm{CP}+\mathrm{P}$ & OS & 12,13 \\
\hline Zhu & 2008 & 731 & 206 & NA & $30(15)$ & $256(35)$ & $146(20)$ & IIIB-IV & $365(50)$ & E vs $P$ & OS & 12,13 \\
\hline Douillard & 2010 & 1466 & 275 & NA & $49(18)$ & $512(34.9)$ & $298(20.3)$ & IIIB-IV & $830(56.6)$ & G vs $D$ & OS & 12,13 \\
\hline Herbst & 2010 & 224 & 88 & 64 & $17(19.3)$ & $99(44.2)$ & NA & IIIB-IV & $128(57.1)$ & $\mathrm{CP}+\mathrm{Cet}$ & OS & 12,13 \\
\hline $\begin{array}{l}\text { Khambata- } \\
\text { Ford }\end{array}$ & -2011 & 676 & 202 & 64.5 & $35(17.3)$ & $280(41.4)$ & $53(7.8)$ & IIIB-IV & NA & $\mathrm{CP}+\mathrm{Cet}$ vs $\mathrm{CP}$ & PFS & 12,13 \\
\hline Brugger & 2011 & 889 & 493 & 60 & $90(18)$ & $231(26)$ & $151(17)$ & IIIB-IV & $400(45)$ & E vs $P$ & PFS 1 & $12,13,61$ \\
\hline O'Byrne & 2011 & 1125 & 395 & NA & 75 (19) & $335(29.8)$ & $244(21.7)$ & IIIB-IV & $532(47.2)$ & CisV+Cet vs CisV & OS & 12,13 \\
\hline Shepherd & 2013 & 3532 & 1543 & NA & $300(19.4)$ & $391(25.3)$ & NA & I-III & $605(39.2)$ & CT vs OBS & OS & $12,13,14$ \\
\hline
\end{tabular}


Table 2. All the Pooled Hazard Ratios of the Meta-analysis

\begin{tabular}{|c|c|c|c|c|c|c|c|}
\hline & & \multicolumn{3}{|c|}{ OS } & \multicolumn{3}{|c|}{ PFS } \\
\hline & & HR $(95 \% \mathrm{CI})$ & $\mathrm{Z}$ & $\mathrm{P}$ & HR $(95 \%$ CI $)$ & $\mathrm{Z}$ & $\mathrm{P}$ \\
\hline \multicolumn{2}{|l|}{ Overall } & $1.39(1.23,1.56)$ & 5.35 & $<0.001$ & $1.33(1,17,1,51)$ & 4.41 & $<0.001$ \\
\hline \multirow[t]{2}{*}{ Disease stage } & Early stage & $1.24(1.03,1.50)$ & 2.32 & 0.02 & & & \\
\hline & Advanced stage & $1.50(1.28,1.76)$ & 5.06 & $<0.001$ & $1.42(1.23,1.64)$ & 4.82 & $<0.001$ \\
\hline \multirow[t]{2}{*}{ Line of therapy } & First line & $1.30(1.13,1.49)$ & 3.69 & $<0.001$ & $1.27(0.99,1.64)^{*}$ & 1.86 & 0.063 \\
\hline & Second- or later-line & $1.94(1.44,2.62)$ & 4.33 & $<0.001$ & $1.60(1.10,2.31)$ & 2.48 & 0.013 \\
\hline \multirow[t]{3}{*}{ Treatment } & chemotherapy & $1.37(1.13,1.66)$ & 3.24 & 0.001 & $1.39(1.08,1.79)$ & 2.52 & 0.012 \\
\hline & EGFR-TKIs & $1.97(1.31,2.95)$ & 3.26 & 0.001 & $1.70(1.27,2,26)$ & 3.61 & $<0.001$ \\
\hline & Chemotherapy+Cet & $1.27(0.92,1.75)$ & 1.47 & 0.141 & $1.14(0.56,1.94)^{*}$ & 0.46 & 0.643 \\
\hline Mutation point & Codon 12,13 & $1.55(1.30,1.84)$ & 4.93 & $<0.001$ & $1.32(1.10,1.57)$ & 3.06 & 0.002 \\
\hline
\end{tabular}

HR, hazard ratio; OS, overall survival; PFS, progression-free survival; EGFR-TKI, epidermal grow factor receptor tyrosine kinase inhibitor; Cet, cetuximab. *The HR was assessed using a DerSimonian-Laird random model

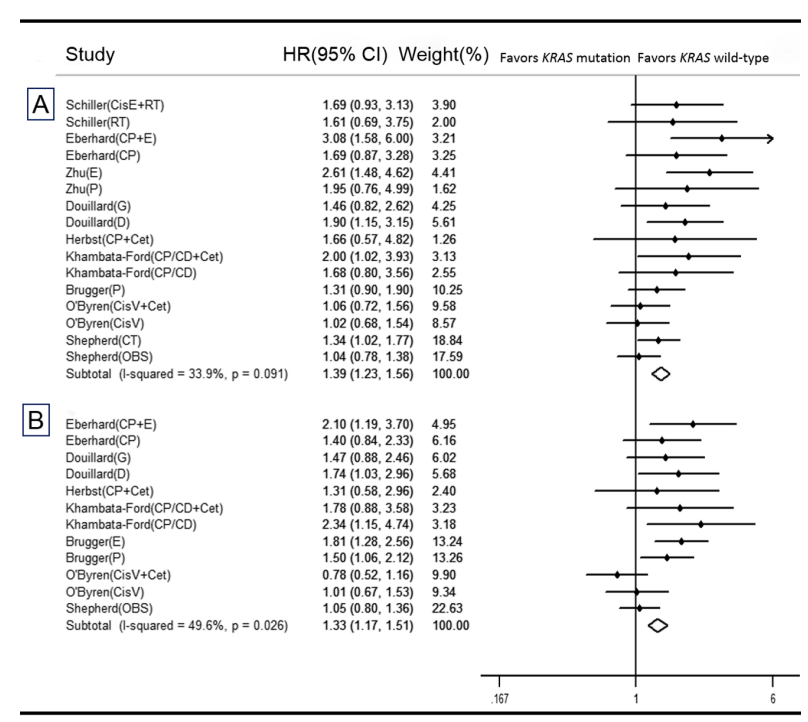

Figure 2. Forest Plots of Pooled HR for OS (A) and PFS (B) Comparing KRAS Mutant Patients with $K R A S$ Wild-Type Patients. HR, hazard ratio; OS, overall survival; PFS, progression-free survival

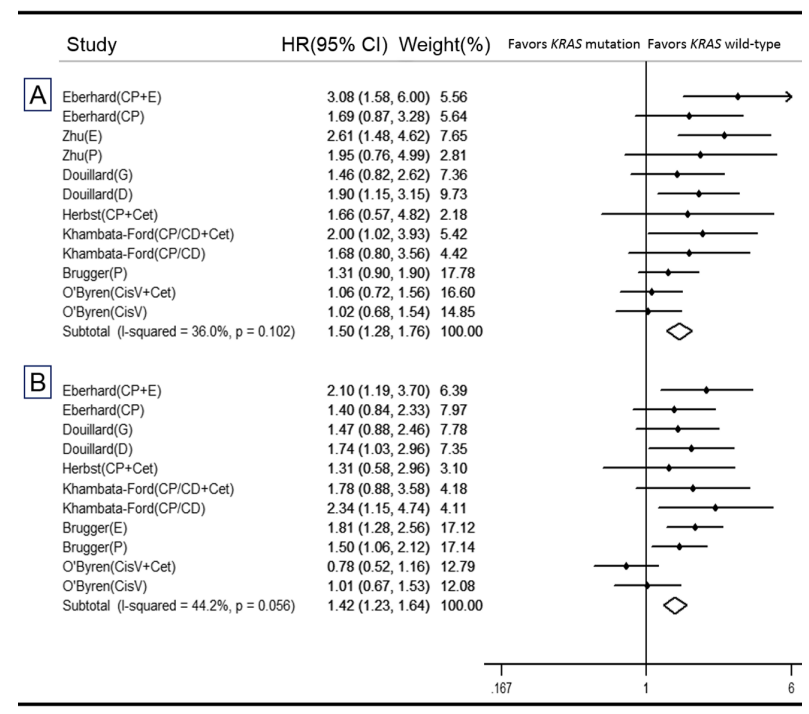

Figure 3. Forest Plots of the Included Studies Using HR for OS (A) and PFS (B) Comparing KRAS Mutant Patients with $K R A S$ wild-type Patients in the Subgroup of Advanced NSCLC Patients. HR, hazard ratio; OS, overall survival; PFS, progression-free survival
Douillard et al., 2010, Herbst et al., 2010, Khambata-Ford et al., 2010, Brugger et al., 2011, O’Byrne et al., 2011, Shepherd et al., 2013) with relevant PFS data included 466 patients with a KRAS mutation and 1938 patients with wild-type $K R A S$. Pooled HR analysis (HR: 1.33; 95\% CI: $1.17-1.51 ; P<0.001$; heterogeneity test $I^{2}=49.6 \%$; $P=0.026)$ indicated a consistent trend toward unfavorable PFS for patients with a KRAS mutation (Figure 2B).

Subgroup analyses according to disease stage, line of therapy, treatment, and point mutation

There were inadequate data for subsequent prespecified analyses for PFS for early stage patients. Following up on the available data, KRAS mutations were associated with poor survival not only for advanced NSCLC according to the pooled HR analysis for OS (HR: $1.50 ; 95 \% \mathrm{CI}$ : 1.28-1.76; $P<0.001$; heterogeneity test $I^{2}=36 \% ; P=0.102$ ) (Figure 3A) and PFS (HR: 1.42; 95\% CI: 1.23-1.64; $P<0.001$; heterogeneity test $I^{2}=44.2 \% ; P=0.056$ ) (Figure 3B) but also for early stage NSCLC based on the overall HR for OS (HR: 1.24; 95\% CI: 1.03-1.50; $\mathrm{P}=0.020$; heterogeneity test $I^{2}=4.2 \% ; P=0.372$ ) (Table 2 ).

Subgroup analyses stratified by line of therapy (Figure 4) were restricted to 6 studies (Schiller et al., 2001, Eberhard et al., 2005; Herbst et al., 2010; Khambata et al., 2010; O'Byrne et al., 2011; Shepherd et al., 2013) for OS and 5 studies (Eberhard et al., 2005; Herbst et al., 2010; Khambata-Ford et al., 2010; O’Byrne et al., 2011; Shepherd et al., 2013) for PFS in which treatments were set for first-line therapy. Significant survival benefits were observed for KRAS wild-type NSCLCs in the OS analysis (HR: 1.30 ; 95\% CI: 1.13-1.49; $P<0.001$; heterogeneity test $I^{2}=33.3 \%$; $P=0.133$ ) (Figure $4 \mathrm{~A}$ ) but not from the results of the PFS analysis (HR: 1.27; 95\% CI: 0.99-1.64; $P=0.063$; heterogeneity test $I^{2}=52.4 \% ; P=0.04$ ) (Figure 4C). However, the presence of a KRAS mutation was a poor prognostic factor for second- or later-line treatment according to the pooled analyses of HR for OS (HR: 1.94; 95\% CI: 1.44-2.62; $P<0.001$; heterogeneity test $I^{2}=0 \%$; $P=0.582$ ) (Figure 4B) and PFS (HR: $1.60 ; 95 \% \mathrm{CI}$ : $1.10-2.31 ; P=0.013$; heterogeneity test $I^{2}=0 \% ; P=0.653$ ) (Figure 4D).

Additional analyses assessing the role of KRAS mutations in the NSCLC subgroup treated with different combinations of therapy were performed by separately aggregating the studies according to chemotherapy, 


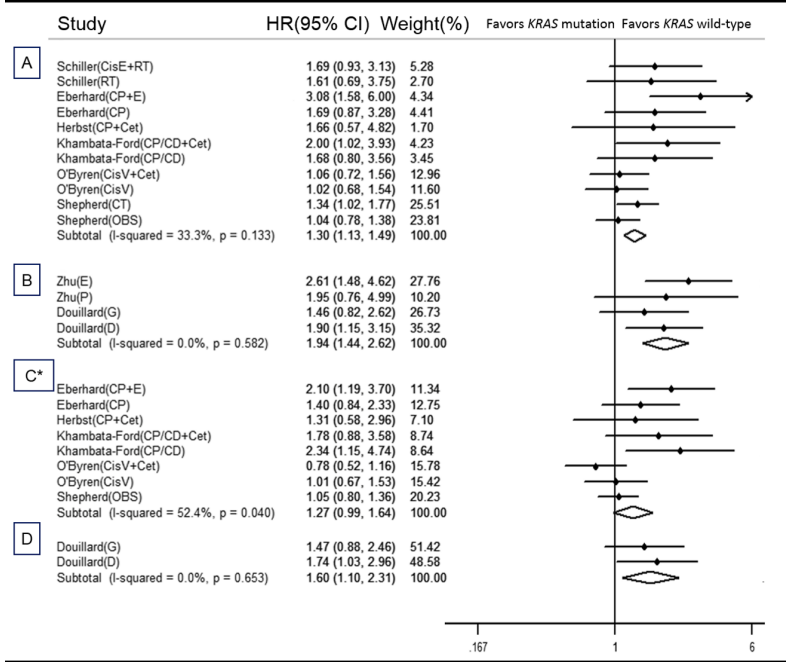

Figure 4. A, B, C and D, Forest Plots of Pooled HR Analyzed According to the Line of Therapy Comparing KRAS Mutant Patients with KRAS Wild-Type Patients. A, OS analysis for patients treated with first line therapy. B, OS analysis for patients treated with second- and higher-line of therapy. C, PFS analysis for patients treated with first line therapy. D, PFS analysis for patients treated with second- and higher-line of therapy. HR, hazard ratio; OS, overall survival; PFS, progression-free survival. *The HR was assessed using a DerSimonian-Laird random model

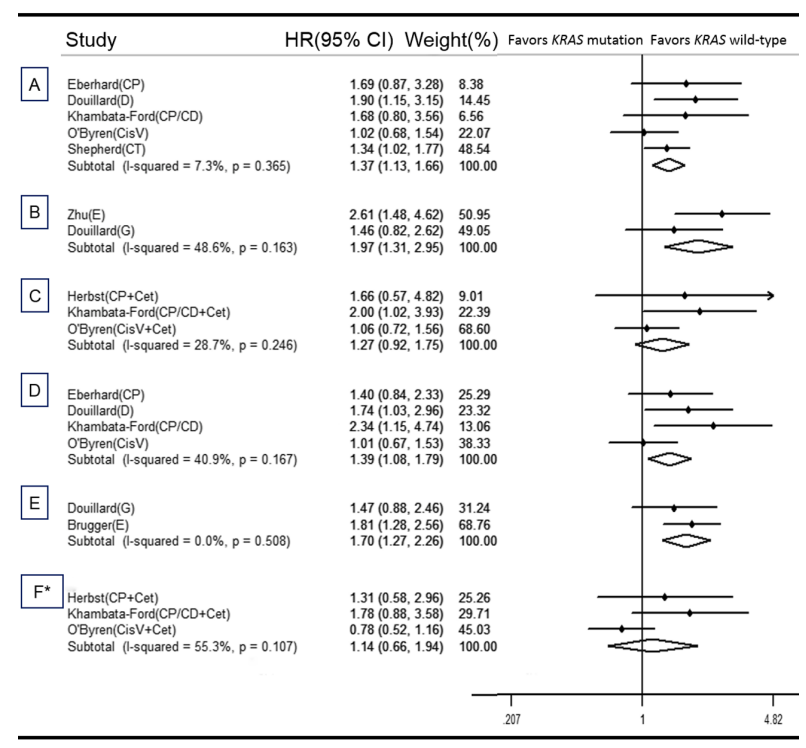

Figure 5. A, B, C, D, E, F, Forest Plots of Pooled HR Comparing KRAS Mutant Patients with KRAS WildType Patients Stratified by the Treatment Regimen. A, OS analysis for patients treated with chemotherapy. B, OS analysis for patients treated with EGFR-TKIs. C, OS analysis for patients treated with chemotherapy coupled with cetuximab. D, PFS analysis for patients treated with chemotherapy. E, PFS analysis for patients treated with EGFR-TKIs. F, PFS analysis for patients treated with chemotherapy coupled with cetuximab. $\mathrm{HR}$, hazard ratio; OS, overall survival; PFS, progression-free survival; EGFR-TKI, epidermal growth factor receptor tyrosine kinase inhibitor. *The HR was assessed using a DerSimonianLaird random mode

EGFR-TKI and chemotherapy coupled with cetuximab. As indicated in Figure 5, there was a statistically significant benefit for OS (HR: 1.37; 95\% CI: 1.13-1.66; $\mathrm{P}=0.001$ )
(Figure 5A) and PFS (HR: 1.39; 95\% CI: 1.08-1.79; $P=0.012$ ) (Figure 5D) for patients with wild-type KRAS tumors when treated with chemotherapy. Similar results were found in the subset of patients treated with EGFRTKIs following the pooled HR estimate for OS (HR: 1.97; 95\% CI: 1.31-2.95; $P=0.001$ ) (Figure 5B) and PFS (HR: 1.70; 95\% CI: 1.27-2.26; $P<0.001$ ) (Figure 5E). However, the pooled HRs for OS (HR: 1.27; 95\% CI: 0.92-1.75; $P=0.141$ ) (Figure 5C) and PFS (HR: 1.14; 95\% CI: 0.661.94; $P=0.643$ ) (Figure $5 \mathrm{~F}$ ) indicated no statistically significant relationship between survival benefit and $K R A S$ status in the NSCLC patient subgroup treated with chemotherapy coupled with cetuximab.

In addition, we conducted an analysis based on data (Eberhard et al., 2005; Zhu et al., 2008; Douillard et al., 2010, Herbst et al., 2010; Khambata-Ford et al., 2010; O'Byrne et al., 2011) from a subgroup of patients harboring KRAS codon 12 or 13 point mutations (Table 2), revealing that the main KRAS mutation category is also related to unfavorable survival in accordance with the combined HRs for OS (HR: 1.55 ; 95\% CI 1.301.84; $P<0.001)$ and PFS (HR: $1.32 ; 95 \%$ CI 1.10-1.57; $P=0.002)$.

\section{Discussion}

Although numerous experimental studies and clinical trials have been conducted over the years, the NSCLC prognosis remains dismal with 5-year overall survival rate of 10-15\% and an even worse survival rate for advancedstage patients (Siegel et al., 2012). The development of basic laboratory findings, genomics and genetic signatures may revolutionize future NSCLC treatment and provide novel insight into future targeted therapies based on the molecular characteristics of individual patient tumors (Schiller et al., 2013). Considering that few validated biomarkers are used to direct clinical treatment decisions, identifying more effective and less toxic treatments is of great importance for the selection of specific patients.

It is not surprising that the presence of a KRAS mutation is a potential biomarkers. In addition to clinical trials, the clinical implication of KRAS mutations was discussed in three meta-analyses (Huncharek et al., 1999; Mascaux et al., 2005; Meng et al., 2013), but there was no clear consensus. In the meta-analysis conducted by Huncharek ${ }^{29}$, the magnitude of the effect of a KRAS mutation was combined with relative risk (RR), indicating a greater chance of death at 2 years from NSCLC, and the results $(\mathrm{RR}=2.35$; 95\% CI 1.61-3.22; heterogeneity test $\mathrm{Q}=15.52 ; P=0.03)$ revealed that $K R A S$ mutation is related to shortened survival. The presence of a KRAS mutation was also confirmed (Mascaux et al., 2005) as a poor prognostic factor (HR: 1.35 ; 95\% CI 1.16-1.56; heterogeneity test $P=0.01)$ using a random model, which may be true for only adenocarcinoma (HR: 1.52; $95 \%$ CI 1.30-1.78) based on a subset analysis assessing the expression of KRAS-p21 (21 kDa protein). When studies were stratified according to stage, a statistically significant impact of KRAS mutation on survival was noted for patients with stage I-IV disease but not those in early stages (stage I or stage I-III). In the latest meta-analysis 
focusing on the role of KRAS mutations (Meng et al., 2013), 41 articles were included ignoring the nature of studies with the publication year ranging from 1990 to 2012. KRAS mutation was suggested as a poor prognostic factor based on the summarized HR for OS (HR: 1.45; 95\% CI 1.29-1.62; heterogeneity test $I^{2}=42.9 \% ; P=0.002$ ) using a random-effects model. Furthermore, similar results were observed in a subgroup analysis based on ethnicity in adenocarcinoma patients and patients harboring a KRAS codon 12 mutation, which strengthens the conclusion that $K R A S$ mutation is a worse prognostic factor for patients with NSCLC. Nevertheless, the combined HRs (HR: 1.30; 95\% CI 0.99-1.71; heterogeneity test $I^{2}=55.3 \% ; P=0.028$ ) for advanced-stage NSCLC indicate no relationship with survival, which differs from the results obtained for early stage patients (stage I or stage I-IIIa disease). As noted above, although the results confirmed that KRAS mutations indicated poor survival benefit in the overall analysis with significant heterogeneity, a discrepancy still exists in the subset analyses regarding disease stage.

Within the 9 articles used in our meta-analysis, the $K R A S$ tumor mutational status was not associated with prognostic benefit for a certain homogeneous group of NSCLC patients according to 6 studies (Schiller et al., 2001; Douillard et al., 2010; Herbst et al., 2010; KhambataFord et al., 2010; O'Byrne et al., 2011; Shepherd et al., 2013). Only one study provided an indication of a lack of survival benefit for patients with KRAS mutations (Zhu et al., 2008). In addition, the association with poor survival benefit was only observed in the analysis of PFS $(P=0.020)$, but it did not correspond with the OS results $(P=0.152)$ in the study reported by Brugger et al. (2011). The study reported by Shepherd et al. (2013), including 4 randomized trials with 1543 patients, was in favor of the idea that KRAS oncogene status is not significantly prognostic for early stage NSCLC although subgroup analysis of patients with a KRAS codon 13 point mutation required more validation. In our meta-analysis based on the best evidence available from randomized trials, we found that KRAS mutation is a poor prognostic factor according to OS and PFS analyses. The poor prognostic role of KRAS mutation exists for early or advanced stage patients and patients treated with chemotherapy or EGFR-TKIs. However, the combine HR for the OS of early stage patients should be interpreted with caution because the results were not particularly overwhelming $(P=0.02)$. When stratifying according to line of therapy, $K R A S$ mutation is still related to poor survival with the exception of the subgroup of patients receiving the chemotherapy as a first-line therapy for the PFS endpoint. However, a trend toward statistical inferiority is noted for $K R A S$ mutant patients $(P=0.06)$. It is noteworthy that no significant relationship is observed for patients treated with chemotherapy coupled with cetuximab, which is a different scenario than that for colorectal cancer patients (Pirker, 2013). This discrepancy may be partially explained by the different pattern of mutational events and complexity of cell signaling pathways in the different tumors. Furthermore, the prognostic ability of $K R A S$ mutations appears to be significantly stronger for OS than PFS based on our results.
To clarify the role of KRAS mutations in NSCLC, we summarized the updated randomized trials in which the $K R A S$ oncogene was assessed according to survival data stratified by mutation status. However, some limitations remain. First, no additional data were acquired from the authors, resulting in the exclusion of some randomized trials due to inadequate data. Second, the exact HRs and 95\% Cls were directly reported for several treatment arms, and some survival data had to be extracted from survival curves, which may be confounded system error and random error. Third, in some treatment arms, the number of KRAS mutant patients was relatively small (less than 20 patients), which increased the possibility of an imbalance in the baseline characteristics and bias within the study. Lastly, the assessment of adverse events is another important factor in making clinical treatment decisions. However, there were no sufficient data for performing analyses in detail to address such a concern.

As indicated above, the presence of a KRAS mutation is associated with poor prognosis for NSCLC, particularly for those with advanced stage disease or received secondor later-line therapy or treated with EGFT-TKIs but not for those treated with chemotherapy in combination with cetuximab.

\section{Acknowledgements}

This study was supported by grants from National Natural Science Foundation of China (NO.81001047/ H1615), Excellent Young Teachers Program of Higher Education of Guangdong Province (Yq2013040), and Research Fund for the Science and technology Star of Zhujiang of Guangzhou City (2014J2200031).

\section{References}

Alimujiang S, Zhang T, Han Z-G, et al (2013). Epidermal growth factor receptor tyrosine kinase inhibitor versus placebo as maintenance therapy for advanced non-small-cell lung cancer: a meta-analysis of randomized controlled trials. Asian Pac J Cancer Prev, 14, 2413-9.

Brugger W, Triller N, Blasinska-Morawiec M, et al (2011). Prospective molecular marker analyses of EGFR and KRAS from a randomized, placebo-controlled study of erlotinib maintenance therapy in advanced non-small-cell lung cancer. J Clin Oncol, 29, 4113-20.

Carney DN (2002). Lung Cancer-time to move on from chemotherapy. N Engl J Med, 346, 126-8.

Chetty R,Govender D (2013). Gene of the month: KRAS. J Clin Pathol, 66, 548-50.

DerSimonian R, Laird N (1986). Meta-analysis in clinical trials. Control Clin Trials, 7, 177-88.

Douillard JY, Shepherd FA, Hirsh V, et al (2010). Molecular predictors of outcome with gefitinib and docetaxel in previously treated non-small-cell lung cancer: data from the randomized phase III INTEREST trial. J Clin Oncol, 28, 744-52.

Eberhard DA, Johnson BE, Amler LC, et al (2005). Mutations in the epidermal growth factor receptor and in KRAS are predictive and prognostic indicators in patients with nonsmall-cell lung cancer treated with chemotherapy alone and in combination with erlotinib. J Clin Oncol, 23, 5900-9.

Goss GD, O'Callaghan C, Lorimer I, et al (2013). Gefitinib 
versus placebo in completely resected non-small-cell lung cancer: results of the NCIC CTG BR19 study. J Clin Oncol, 31, 3320-6.

Graziano SL, Gamble GP, Newman NB, et al (1999). Prognostic significance of K-ras codon 12 mutations in patients with resected stage I and II non-small-cell lung cancer. J Clin Oncol, 17, 668-75.

Herbst RS, Kelly K, Chansky K, et al (2010). Phase II selection design trial of concurrent chemotherapy and cetuximab versus chemotherapy followed by cetuximab in advancedstage non-small-cell lung cancer: Southwest oncology group study S0342. J Clin Oncol, 28, 4747-54.

Higgins JP, Thompson SG, Deeks JJ, Altman DG (2003). Measuring inconsistency in meta-analyses. $B M J, 327$, 557-60.

Huncharek M, Muscat J,Geschwind JF (1999). K-ras oncogene mutation as a prognostic marker in non-small cell lung cancer: a combined analysis of 881 cases. Carcinogenesis, 20, 1507-10.

Johnson ML, Sima CS, Chaft J, et al (2013). Association of KRAS and EGFR mutations with survival in patients with advanced lung adenocarcinomas. Cancer, 119, 356-62.

Khambata-Ford S, Harbison CT, Hart LL, et al (2010). Analysis of potential predictive markers of cetuximab benefit in BMS099, a phase III study of cetuximab and first-line taxane/ carboplatin in advanced non-small-cell lung cancer. J Clin Oncol, 28, 918-27.

Liberati A, Altman DG, Tetzlaff J, et al (2009). The PRISMA statement for reporting systematic reviews and metaanalyses of studies that evaluate health care interventions: explanation and elaboration. Ann Intern Med, 151, 65-94.

Mantel N, Haenszel W (1959). Statistical aspects of the analysis of data from retrospective. J Natl Cancer Inst, 22, 719-48.

Martin P, Leighl NB, Tsao MS, Shepherd FA (2013). KRAS mutations as prognostic and predictive markers in non-small cell lung cancer. $J$ Thorac Oncol, 8, 530-42.

Mascaux C, Iannino N, Martin B, et al (2005). The role of RAS oncogene in survival of patients with lung cancer: a systematic review of the literature with meta-analysis. $\mathrm{Br} \mathrm{J}$ Cancer, 92, 131-9.

Meng D, Yuan M, Li X, et al (2013). Prognostic value of K-RAS mutations in patients with non-small cell lung cancer: a systematic review with meta-analysis. Lung Cancer, 81, $1-10$.

NCCN (2012). Clinical practice guidelines in oncology (NCCN Guidelines ${ }^{\circledR}$ ): non-small cell lung cancer. Cochrane Database of Systematic Reviews 2014.

O’Byrne KJ, Gatzemeier U, Bondarenko I, et al (2011). Molecular biomarkers in non-small-cell lung cancer: a retrospective analysis of data from the phase 3 FLEX study. Lancet Oncol, 13, 795-805.

Pirker R (2013). EGFR-directed monoclonal antibodies in nonsmall cell lung cancer. Target Oncol, 8, 47-53.

Qi W-X, Shen Z, Lin F, et al (2012). Comparison of the efficacy and safety of EFGR tyrosine kinase inhibitor monotherapy with standard second-line chemotherapy in previously treated advanced non-small-cell lung cancer: a systematic review and meta-analysis. Asian Pac J Cancer Prev, 13, 5177-82.

Ramalingam SS, Owonikoko TK, Khuri FR (2011). Lung cancer: new biological insights and recent therapeutic advances. $C A$ Cancer J Clin, 61, 91-112.

Riely GJ, Kris MG, Rosenbaum D, et al (2008). Frequency and distinctive spectrum of $K R A S$ mutations in never smokers with lung adenocarcinoma. Clin Cancer Res, 14, 5731-4.

Roberts PJ, Stinchcombe TE (2014). KRAS mutation: should we test for it, and does it matter? J Clin Oncol, 31, 1112-57.
Santos E, Martin-Zanca D, Reddy EP, et al (1984). Malignant activation of a K-ras oncogene in lung carcinoma but not in normal tissue of the same patient. Science, 223, 661-4.

Schiller JH, Adak S, Feins RH, et al (2001). Lack of prognostic significance of $\mathrm{p} 53$ and $\mathrm{K}$-ras mutations in primary resected non-small-cell lung cancer on E4592: A laboratory ancillary study on an eastern cooperative oncology group prospective randomized trial of postoperative adjuvant therapy. J Clin Oncol, 19, 448-57.

Schiller JH, Gandara DR, Goss GD, Vokes EE (2013). Non-smallcell lung cancer: then and now. J Clin Oncol, 31, 981-3.

Shepherd FA, Domerg C, Hainaut P, et al (2013). Pooled analysis of the prognostic and predictive effects of $K R A S$ mutation status and KRAS mutation subtype in early-stage resected non-small-cell lung cancer in four trials of adjuvant chemotherapy. J Clin Oncol, 31, 2173-81.

Siegel R, Naishadham D, Jemal A (2012). Cancer statistics, 2012. CA Cancer J Clin, 62, 10-29.

Slebos RJ, Kibbelaar RE, Dalesio O, et al (1990). K-ras oncogene activation as a prognostic marker in adenocarcinoma of the lung. N Engl J Med, 323, 561-5.

Tierney JF, Stewart LA, Ghersi D, Burdett S, Sydes MR (2007). Practical methods for incorporating summary time-to-event data into meta-analysis. Trials, $\mathbf{8}, 16$.

Usuda K, Sagawa M, Motono N, et al (2014). Relationships between EGFR Mutation status of lung cancer and preoperative factors-are they predictive? Asian Pac J Cancer Prev, 15, 657-62.

Zhu CQ, da Cunha Santos G, Ding K, et al (2008). Role of KRAS and EGFR as biomarkers of response to erlotinib in national cancer institute of canada clinical trials group study BR.21. J Clin Oncol, 26, 4268-75. 